\title{
Restricted Radon Transforms and Unions of Hyperplanes
}

\section{Daniel M. Oberlin}

\begin{abstract}
We study $L^{p}\left(\mathbb{R}^{n}\right) \rightarrow L_{d \mu(\sigma)}^{\alpha, \infty}\left(L_{d t}^{\infty}\right)$ estimates for the Radon transform in certain cases where the dimension of the measure $\mu$ on $\Sigma^{(n-1)}$ is less than $n-1$.
\end{abstract}

\section{Introduction}

If $\Sigma^{(n-1)}$ is the unit sphere in $\mathbb{R}^{n}$, the Radon transform $R f$ of a suitable function $f$ on $\mathbb{R}^{n}$ is defined by

$$
R f(\sigma, t)=\int_{\sigma^{\perp}} f(p+t \sigma) d m_{n-1}(p) \quad \sigma \in \Sigma^{(n-1)}, t \in \mathbb{R},
$$

where the integral is with respect to $(n-1)$-dimensional Lebesgue measure on the hyperplane $\sigma^{\perp}$. We also define, for $0<\delta<1$,

$$
R_{\delta} f(\sigma, t)=\delta^{-1} \int_{\left[\sigma^{\perp} \cap B(0,1)\right]+B(0, \delta)} f(x+t \sigma) d m_{n}(x) .
$$

The paper [5] contains the sharp mapping properties of $R$ from $L^{p}\left(\mathbb{R}^{n}\right)$ into the mixed norm spaces defined by the norms

$$
\|g\|_{L^{q}\left(L^{r}\right)}=\left(\int_{\Sigma^{(n-1)}}\left[\int_{-\infty}^{\infty}|g(\sigma, t)|^{r} d t\right]^{q / r} d \sigma\right)^{1 / q} .
$$

Here $d \sigma$ denotes Lebesgue measure on $\Sigma^{(n-1)}$. The purpose of this paper is mainly to study the possibility of analogous mixed norm estimates when $d \sigma$ is replaced by measures $d \mu(\sigma)$ supported on subsets $S \subseteq \Sigma^{(n-1)}$ having 
dimension $<n-1$. We are usually interested in the case $r=\infty$ and will mostly settle for estimates of restricted weak type in the indices $p$ and $q$ and those only for $f$ supported in a ball. The following theorem, which we regard as an estimate for a restricted Radon transform, is typical of our results here:

Theorem 1. Fix $\alpha \in(1, n-1)$. Suppose $\mu$ is a nonnegative Borel measure on $\Sigma^{(n-1)}$ satisfying the Frostman condition

$$
\int_{\Sigma^{(n-1)}} \int_{\Sigma^{(n-1)}} \frac{d \mu\left(\sigma_{1}\right) d \mu\left(\sigma_{2}\right)}{\left|\sigma_{1}-\sigma_{2}\right|^{\alpha}}<\infty .
$$

Then, for some $C=C(n, \alpha, \mu)$,

$$
\lambda \mu\left(\left\{\sigma \in \Sigma^{(n-1)}: \sup _{t \in \mathbb{R}} R \chi_{E}(\sigma, t)>\lambda\right\}\right)^{1 / \alpha} \leq C|E|^{1 / 2}
$$

for $\lambda>0$ and Borel $E \subseteq B(0,1)$. That is,

$$
\left\|R \chi_{E}\right\|_{L_{\mu}^{\alpha, \infty}\left(L^{\infty}\right)} \leq C|E|^{1 / 2} \text {. }
$$

Suppose that $\alpha \in(0, n-1)$. Say that a Borel set $E \subseteq \mathbb{R}^{n}$ satisfies the (Besicovitch) condition $B(n-1 ; \alpha)$ if there is a compact set $S \subseteq \Sigma^{(n-1)}$ having Hausdorff dimension $\alpha$ such that for each $\sigma \in S$ there is a translate of an $(n-1)$-plane orthogonal to $\sigma$ which intersects $E$ in a set of positive $(n-1)$-dimensional Lebesgue measure.

It is well-known that, given $\epsilon \in(0, \alpha)$, such an $S$ supports a probability measure $\mu$ satisfying the hypothesis of Theorem 1 , but with $\alpha-\epsilon$ in place of $\alpha$. If $\alpha>1$, Theorem 1, in conjunction with standard arguments, implies that such an $E$ must have positive $n$-dimensional Lebesgue measure. That is, $B(n-1 ; \alpha)$ sets in $\mathbb{R}^{n}$ have positive Lebesgue measure if $\alpha>1$.

As will be pointed out in $\S 2$, the next theorem implies that, for $\alpha \in(0,1)$ and in certain cases, $B(n-1 ; \alpha)$ sets have Hausdorff dimension at least $n-1+\alpha$. (Here is a notational comment: $|E|$ will usually denote the Lebesgue measure of $E$ with the appropriate dimension being clear from the context.)

Theorem 2. Suppose $\alpha \in(0,1)$. Suppose $\widetilde{\mu}$ is a nonnegative measure on a compact interval $J \subseteq \mathbb{R}$ which satisfies the condition

$$
\widetilde{\mu}(I) \leq C(\widetilde{\mu})|I|^{\alpha}
$$

for subintervals $I \subseteq J$. Let $\mu$ be the image of $\widetilde{\mu}$ under a one-to-one and bi-Lipschitz mapping of $J$ into $\Sigma^{(n-1)}$. If $0<\gamma<\beta<\alpha$ and

$$
\frac{1}{p}=\frac{1+\beta-\gamma}{1+2 \beta-\gamma}, \quad \frac{1}{q}=\frac{1+\gamma}{1+2 \beta-\gamma}, \quad \eta=\frac{1-\gamma}{1+2 \beta-\gamma}
$$


then there is the estimate

$$
\left\|R_{\delta} \chi_{E}\right\|_{L_{\mu}^{q, \infty}\left(L^{\infty}\right)} \leq C|E|^{1 / p} \delta^{-\eta}
$$

for $C=C(n, \mu, \alpha, \beta, \gamma)$ and for all Borel $E \subset B(0,1)$ and $\delta \in(0,1)$.

Contrasting with Theorems 1 and 2, the next result provides a global estimate for a restricted Radon transform:

Theorem 3. Suppose $n \geq 4$. Let $S$ be the $(n-2)$-sphere

$$
\left\{\sigma=\left(\sigma_{1}, \ldots, \sigma_{n}\right) \in \Sigma^{(n-1)}: \sum_{1}^{n-1} \sigma_{j}^{2}=\sigma_{n}^{2}\right\}
$$

and let $\mu$ be Lebesgue measure on $S$. Then there is an estimate

$$
\left\|R \chi_{E}\right\|_{L_{\mu}^{n-2}\left(L^{\infty}\right)} \leq C|E|^{(n-1) / n}
$$

for $C=C(n)$ and for all Borel $E \subseteq \mathbb{R}^{n}$.

Of course it follows, as in the remark after Theorem 1, that if a Borel set $E \subseteq \mathbb{R}^{n}$ has the property that for each $\sigma$ in the $(n-2)$-sphere $S$ there is a translate of an $(n-1)$-plane orthogonal to $\sigma$ which intersects $E$ in a set of positive $(n-1)$-dimensional Lebesgue measure, then $E$ has positive $n$-dimensional Lebesgue measure. Theorem 3 is an analogue of (3) in [5] (which is a similar estimate but with $\mu$ replaced by Lebesgue measure on $\Sigma^{(n-1)}$ and $\left.q=n\right)$. The proof of Theorem 3 parallels the proof in [5] but requires the $L^{2}$ Fourier restriction estimates for the light cone in $\mathbb{R}^{n}$ in place of an easier $L^{2}$ estimate used in [5].

The main method employed in this paper is elementary and reasonably flexible, but it does not yield sharp results. For example, if $n \geq 3$ and if $\mu$ is Lebesgue measure on $\Sigma^{(n-1)}$, then Theorem 1 gives an $L^{2,1}\left(\mathbb{R}^{n}\right) \rightarrow$ $L_{\mu}^{\alpha, \infty}\left(L^{\infty}\right)$ estimate for $1 \leq \alpha<n-1$, while the sharp estimate (from [5]) is $L^{n /(n-1), 1}\left(\mathbb{R}^{n}\right) \rightarrow L_{\mu}^{n}\left(L^{\infty}\right)$. In particular, it seems likely that Theorem 2 holds for general $\alpha$-dimensional measures $(0<\alpha<1)$. (We have some unpublished partial results in this direction for measures of Cantor type.)

The remainder of this paper is organized as follows: $\S 2$ contains the proofs of Theorems 2 and 3 and the statement and proof of a similar result in the case when $d$ is an integer strictly between 1 and $n-1$ and $\mu$ is Lebesgue measure on a suitable $d$-manifold in $\Sigma^{(n-1)} ; \S 3$ contains the proof of Theorem 3; $\S 4$ contains some miscellaneous observations and remarks: an analogue for Kahane's notion of Fourier dimension of Theorem 2 when $n=2$; three examples bearing on the question of whether $B(2 ; 1)$ sets in $\mathbb{R}^{3}$ must have positive measure or only full dimension (the answer depends on the set $S$ of directions); and some comments relating the size of $\cup_{P \in \mathcal{P}} P$ to the size of $\mathcal{P}$ when $\mathcal{P}$ is a collection of hyperplanes in $\mathbb{R}^{n}$. 


\section{Proofs of Theorems 1 and 2}

As Theorem 1 is a consequence of its analogue, uniform in $\delta \in(0,1)$, for the operators $R_{\delta}$, we will restrict our attention to these operators. A standard method for obtaining restricted weak type estimates is to estimate $|E|$ from below. We will do this with a particularly simple-minded strategy based on two observations and originally employed in [3] and [4]. (The paper [3] contains a not-quite-sharp estimate for the Radon transform when $n=2$ and was partial motivation for [5], while [4] contains estimates for a restricted $\mathrm{X}$-ray transform in $\mathbb{R}^{n}$ for $n \geq 3$.) The first observation is that

$$
\left|\cup_{n=1}^{N} E_{n}\right| \geq \sum_{n=1}^{N}\left|E_{n}\right|-\sum_{1 \leq m<n \leq N}\left|E_{m} \cap E_{n}\right| .
$$

The second is the well-known fact that if $\sigma \in \Sigma^{(n-1)}$ and if, for $t \in \mathbb{R}, P_{\sigma}^{\delta}$ denotes a plate $\left[\sigma^{\perp} \cap B(0,1)\right]+B(0, \delta)+t \sigma$, then

$$
\left|P_{\sigma_{1}}^{\delta} \cap P_{\sigma_{2}}^{\delta}\right| \leq \frac{C(n) \delta^{2}}{\left|\sigma_{1}-\sigma_{2}\right|}
$$

(so long as $\sigma_{1}$ and $\sigma_{2}$ are not too far apart, an hypothesis we tacitly assume since it can be achieved by multiplying the measures $\mu$ appearing below by an appropriate partition of unity). Thus if, for $n=1, \ldots, N$, we have plates $P_{\sigma_{n}}^{\delta}$ satisfying $\left|E \cap P_{\sigma_{n}}^{\delta}\right| \geq C_{1} \lambda \delta$, it follows that

$$
|E| \geq C_{1} N \lambda \delta-C(n) \delta^{2} \sum_{1 \leq m<n \leq N} \frac{1}{\left|\sigma_{m}-\sigma_{n}\right|} .
$$

Our strategy, then, will be to choose $N$ and

$$
\sigma_{n} \in\left\{\sigma \in \Sigma^{(n-1)}: \sup _{t \in \mathbb{R}} R_{\delta} \chi_{E}(\sigma, t)>\lambda\right\}
$$

so that (2) gives, for example,

$$
|E| \gtrsim \lambda^{2} \mu\left(\left\{\sigma \in \Sigma^{(n-1)}: \sup _{t \in \mathbb{R}} R_{\delta} \chi_{E}(\sigma, t)>\lambda\right\}\right)^{2 / \alpha},
$$

which is the analogue of (1) for the operator $R_{\delta}$. For Theorem 1 the following lemma will facilitate this choice:

Lemma 1. Let $\mu$ be as in Theorem 1. There is $C=C(\mu)$ such that given $n \in \mathbb{N}$ and a Borel $S \subseteq \Sigma^{(n-1)}$ with $\mu(S)>0$, one can choose $\sigma_{n} \in S$, $1 \leq n \leq N$, such that

$$
\sum_{1 \leq m<n \leq N} \frac{1}{\left|\sigma_{m}-\sigma_{n}\right|} \leq \frac{C N^{2}}{\mu(S)^{2 / \alpha}} .
$$


Proof of Lemma 1. Suppose $\sigma_{1}, \ldots, \sigma_{N}$ are chosen independently and at random from the probability space $\left(S, \frac{\mu}{\mu(S)}\right)$. Then, for $1 \leq m<n \leq N$,

$$
\begin{aligned}
& \mathbb{E}\left(\frac{1}{\left|\sigma_{m}-\sigma_{n}\right|}\right)=\frac{1}{\mu(S)^{2}} \int_{S} \int_{S} \frac{1}{\left|\sigma_{m}-\sigma_{n}\right|} d \mu\left(\sigma_{m}\right) d \mu\left(\sigma_{n}\right) \\
& \leq \frac{1}{\mu(S)^{2}}\left(\int_{S} \int_{S} 1 d \mu\left(\sigma_{m}\right) d \mu\left(\sigma_{n}\right)\right)^{1-1 / \alpha}\left(\int_{S} \int_{S} \frac{1}{\left|\sigma_{m}-\sigma_{n}\right|^{\alpha}} d \mu\left(\sigma_{m}\right) d \mu\left(\sigma_{n}\right)\right)^{1 / \alpha} \\
& \leq \frac{C}{\mu(S)^{2 / \alpha}}
\end{aligned}
$$

by the hypothesis on $\mu$. Thus

$$
\mathbb{E}\left(\sum_{1 \leq m<n \leq N} \frac{1}{\left|\sigma_{m}-\sigma_{n}\right|}\right) \leq \frac{C N^{2}}{\mu(S)^{2 / \alpha}}
$$

and the lemma follows.

Proof of Theorem 1. Let $S$ be the set

$$
\left\{\sigma \in \Sigma^{(n-1)}: \sup _{t \in \mathbb{R}} R_{\delta} \chi_{E}(\sigma, t)>\lambda\right\}
$$

so that if $\sigma \in S$ then there is $t \in \mathbb{R}$ such that if

$$
P_{\sigma}^{\delta}=\left[\sigma^{\perp} \cap B(0,1)\right]+B(0, \delta)+t \sigma
$$

then $\left|E \cap P_{\sigma}^{\delta}\right| \geq C_{1} \lambda \delta$. The conjunction of Lemma 1 and (2) yields

$$
|E| \geq C_{1} N \lambda \delta-C_{2} \delta^{2} N^{2} \mu(S)^{-2 \alpha} .
$$

We consider two cases (noting that $N=N_{0} \doteq \lambda C_{1} \mu(S)^{2 / \alpha} / C_{2} \delta$ makes the RHS of (3) equal to 0 ):

Case I: Assume $N_{0}>10$.

In this case choose $N \in \mathbb{N}$ such that

$$
\frac{\lambda C_{1} \mu(S)^{2 / \alpha}}{2 C_{2} \delta} \geq N \geq \frac{\lambda C_{1} \mu(S)^{2 / \alpha}}{3 C_{2} \delta} .
$$

Then it follows from (3) that

$$
|E| \geq C_{1} \frac{\lambda C_{1} \mu(S)^{2 / \alpha}}{3 C_{2} \delta} \lambda \delta-C_{2} \delta^{2} \frac{\lambda^{2} C_{1}^{2} \mu(S)^{4 / \alpha}}{4 C_{2}^{2} \delta^{2}} \mu(S)^{-2 / \alpha}=\kappa \lambda^{2} \mu(S)^{2 / \alpha}
$$

for $\kappa=C_{1}^{2} /\left(12 C_{2}\right)$. This gives

$$
\lambda \mu(S)^{1 / \alpha} \lesssim|E|^{1 / 2}
$$

as desired. 
Case II: Assume $N_{0} \leq 10$.

In this case (unless $S$ is empty) we estimate

$$
|E| \geq C_{1} \lambda \delta \geq \frac{\lambda^{2} C_{1}^{2} \mu(S)^{2 / \alpha}}{10 C_{2}}
$$

which again yields $\lambda \mu(S)^{1 / \alpha} \lesssim|E|^{1 / 2}$ and so completes the proof of Theorem 1 .

The proof of Theorem 2 requires an analogue of Lemma 1:

Lemma 2. Suppose $\mu$ is as in Theorem 2. Suppose $0<\gamma<\beta<\alpha$. Then there is $C=C(\alpha, \mu, \beta, \gamma)$ such that given a Borel $S \subseteq \Sigma^{(n-1)}$ with $\mu(S)>0$ and $N \in \mathbb{N}$, one can choose $\sigma_{n} \in S, 1 \leq n \leq N$, such that

$$
\sum_{1 \leq m<n \leq N} \frac{1}{\left|\sigma_{m}-\sigma_{n}\right|} \leq \frac{C N^{(1+2 \beta-\gamma) / \beta}}{\mu(S)^{(1+\gamma) / \beta}} .
$$

Proof of Lemma 2. It suffices to show that there exists $C$ such that if $F$ is a measurable subset of $J$ with $\widetilde{\mu}(F)>0$ and if $N \in 2 \mathbb{N}$, then there are $x_{1}, \ldots, x_{N / 2}$ in $F$ such that

$$
\sum_{1 \leq m<n \leq N / 2} \frac{1}{\left|x_{m}-x_{n}\right|} \leq \frac{C N^{(1+2 \beta-\gamma) / \beta}}{\widetilde{\mu}(F)^{(1+\gamma) / \beta}} .
$$

Note that because $\beta<\alpha$ it follows that $\widetilde{\mu}(I) \lesssim|I|^{\beta}$ for subintervals $I$ of $J$. Now define $\eta$ by $\eta^{\beta}=\widetilde{\mu}(F) / N$ and find $a_{1}<b_{1} \leq a_{2}<\cdots<b_{N}$ in $J$ such that $\widetilde{\mu}\left(F \cap\left[a_{n}, b_{n}\right]\right)=\eta^{\beta}$. Let $I_{n}=\left[a_{n}+\eta / L, b_{n}-\eta / L\right]$ where $L$ is chosen large enough to guarantee that $\widetilde{\mu}\left(F \cap I_{n}\right) \geq \eta^{\beta} / 2$ and then find intervals $\widetilde{I}_{n} \subseteq I_{n}$ satisfying $\widetilde{\mu}\left(F \cap \widetilde{I}_{n}\right)=\eta^{\beta} / 2$. Choose Borel mappings

$$
\tau_{n}:\left[0, \eta^{\beta} / 2\right] \rightarrow F \cap \widetilde{I}_{n}
$$

such that the equalities

$$
\int_{F \cap \widetilde{I_{n}}} f d \widetilde{\mu}=\int_{0}^{\eta^{\beta} / 2} f\left(\tau_{n}(s)\right) d m_{1}(s)
$$

hold for reasonable functions $f$ on $F \cap \widetilde{I}_{n}$. Then

$$
\int_{0}^{\eta^{\beta} / 2} \int_{0}^{\eta^{\beta} / 2} \sum_{n \neq m} \frac{d m_{1}(s) d m_{1}(t)}{\left|\tau_{m}(s)-\tau_{n}(t)\right|}=\sum_{n \neq m} \int_{F \cap \widetilde{I_{m}}} \int_{F \cap \widetilde{I_{n}}} \frac{d \widetilde{\mu}(x) d \widetilde{\mu}(y)}{|x-y|} .
$$


Since $\gamma<1$ and $d\left(\widetilde{I_{m}}, \widetilde{I_{n}}\right) \geq \eta / L$, the last sum is

$$
\begin{aligned}
& \leq C \eta^{\gamma-1} \int_{F} \int_{F} \frac{d \widetilde{\mu}(x) d \widetilde{\mu}(y)}{|x-y|^{\gamma}} \\
& \leq C \eta^{\gamma-1}\left(\int_{F} \int_{F} \frac{d \widetilde{\mu}(x) d \widetilde{\mu}(y)}{|x-y|^{\beta}}\right)^{\gamma / \beta} \widetilde{\mu}(F)^{2(1-\gamma / \beta)} \\
& =C \eta^{\gamma-1} \widetilde{\mu}(F)^{2(1-\gamma / \beta)}
\end{aligned}
$$

since

$$
\int_{J} \int_{J} \frac{d \widetilde{\mu}(x) d \widetilde{\mu}(y)}{|x-y|^{\beta}}<\infty
$$

follows from the hypothesis on $\widetilde{\mu}$ and the fact that $\beta<\alpha$. Thus

$$
\begin{aligned}
\frac{1}{\left(\eta^{\beta} / 2\right)^{2}} & \int_{0}^{\eta^{\beta} / 2} \int_{0}^{\eta^{\beta} / 2} \sum_{n \neq m} \frac{d m_{1}(s) d m_{1}(t)}{\left|\tau_{m}(s)-\tau_{n}(t)\right|} \leq C \eta^{-2 \beta+\gamma-1} \widetilde{\mu}(F)^{2(1-\gamma / \beta)} \\
& =C\left(\frac{\widetilde{\mu}(F)}{N}\right)^{(-2 \beta+\gamma-1) / \beta} \widetilde{\mu}(F)^{2(1-\gamma / \beta)}=C N^{(2 \beta-\gamma+1) / \beta} \widetilde{\mu}(F)^{-(1+\gamma) / \beta} .
\end{aligned}
$$

It follows that there are $s, t \in\left[0, \eta^{\beta} / 2\right]$ such, for $m, n=1, \ldots, N$, the points

$$
x_{n}=\tau_{n}(s) \in F \cap \widetilde{I_{n}}, \quad y_{m}=\tau_{m}(t) \in F \cap \widetilde{I_{m}}
$$

satisfy

$$
\sum_{n \neq m} \frac{1}{\left|x_{m}-y_{n}\right|} \leq \frac{C N^{(2 \beta-\gamma+1) / \beta}}{\widetilde{\mu}(F)^{(1+\gamma) / \beta}} .
$$

Now either $x_{n} \leq y_{n}$ for at least $N / 2 n$ 's or $y_{n} \leq x_{n}$ for at least $N / 2 n$ 's. Without loss of generality, consider the first case and let

$$
\mathcal{N}=\left\{n=1, \ldots, N: x_{n} \leq y_{n}\right\} .
$$

If $n_{1}, n_{2} \in \mathcal{N}$ and $n_{1}<n_{2}$ then (because $y_{n_{1}} \in I_{n_{1}}$ and $x_{n_{2}} \in I_{n_{2}}$ ), we have

$$
x_{n_{1}} \leq y_{n_{1}}<x_{n_{2}} \leq y_{n_{2}}
$$

and so

$$
\left|x_{n_{1}}-x_{n_{2}}\right|>\left|y_{n_{1}}-x_{n_{2}}\right| .
$$

Thus

$$
\begin{aligned}
\sum_{\substack{n_{1}<n_{2} \\
\rightarrow} n_{1}, n_{2} \in \mathcal{N}} \frac{1}{\left|x_{n_{1}}-x_{n_{2}}\right|} & <\sum_{\substack{n_{1}<n_{2} \\
\rightarrow}} \frac{1}{\mid y_{1}, n_{2} \in \mathcal{N}}-x_{n_{1}} \mid \\
& \leq \sum_{n \neq m} \frac{1}{\left|x_{m}-y_{n}\right|} \leq \frac{C N^{(2 \beta-\gamma+1) / \beta}}{\widetilde{\mu}(F)^{(1+\gamma) / \beta}}
\end{aligned}
$$

Renumbering a subset of $\left\{x_{n}\right\}_{n \in \mathcal{N}}$ gives (4) and completes the proof of the lemma. 
Proof of Theorem 2. The proof is parallel to that of Theorem 1. Using Lemma 2 instead of Lemma 1, the analogue of (3) is

$$
|E| \geq C_{1} N \lambda \delta-C_{2} \delta^{2} N^{(1+2 \beta-\gamma) / \beta} \mu(S)^{-(1+\gamma) / \beta} .
$$

The two cases are now defined by comparing

$$
N_{0} \doteq\left(\frac{C_{1} \lambda}{C_{2} \delta}\right)^{\beta /(1+\beta-\gamma)} \mu(S)^{\frac{1+\gamma}{1+\beta-\gamma}}
$$

and 10. In case $N_{0}>10$, choosing $N$ in (5) such that $N_{0} / 2 \geq N \geq N_{0} / 3$ gives

$$
|E| \geq \lambda^{\frac{1+2 \beta-\gamma}{1+\beta-\gamma}} \delta^{\frac{1-\gamma}{1+\beta-\gamma}} \mu(S)^{\frac{1+\gamma}{1+\beta-\gamma}} \kappa
$$

where

$$
\kappa=C_{1}^{\frac{1+2 \beta-\gamma}{1+\beta-\gamma}} C_{2}^{\frac{-\beta}{1+\beta-\gamma}}\left(\frac{1}{3}-\frac{1}{2^{(1+2 \beta-\gamma) / \beta}}\right)>0 .
$$

This leads directly to the desired estimate $\lambda \mu(S)^{1 / q} \lesssim|E|^{1 / p} \delta^{-\eta}$ if $N_{0}>10$.

On the other hand, the inequality $N_{0} \leq 10$ gives $\lambda \mu(S)^{(1+\gamma) / \beta} \lesssim \delta$ and so

$$
\lambda^{A} \mu(S)^{A(1+\gamma) / \beta} \lesssim \delta^{A}
$$

if $A>0$. Since $|E| \geq C_{1} \lambda \delta$ (unless $S$ is empty), there is also the inequality

$$
\lambda^{1-A} \lesssim|E|^{1-A} \delta^{A-1}
$$

as long as $0<A<1$. Multiplying (6) and (7) gives

$$
\lambda \mu(S)^{A(1+\gamma) / \beta} \lesssim|E|^{1-A} \delta^{2 A-1} .
$$

Then the choice $A=\beta /(1+2 \beta-\gamma)$ yields $\lambda \mu(S)^{1 / q} \lesssim|E|^{1 / p} \delta^{-\eta}$ again, completing the proof of Theorem 2 .

It follows from a small modification of the proof of Lemma 2.15 in [1] that the estimate

$$
\left\|R_{\delta} \chi_{E}\right\|_{L_{\mu}^{q, \infty}\left(L^{\infty}\right)} \lesssim|E|^{1 / p} \delta^{-\eta}
$$

implies a lower bound of $n-p \eta$ for the Hausdorff dimension of a Borel set containing positive-measure sections of hyperplanes associated with each of the directions $\sigma$ in the support of $\mu$. Plugging in the values for $p$ and $\eta$ which are given in Theorem 2 yields first the lower bound $n-(1-\gamma) /(1+\beta-\gamma)$ and then, since that is valid for $0<\gamma<\beta<\alpha$, the desired lower bound of $n-1+\alpha$. A subset $S \subseteq \Sigma^{(n-1)}$ of Hausdorff dimension $\alpha \in(0,1)$ and located on a curve as in the hypotheses of Theorem 2 , will, for each $\epsilon \in(0, \alpha)$, 
support a measure $\mu$ satisfying the hypotheses of Theorem 2 , but with $\alpha-\epsilon$ instead of $\alpha$. It follows that the $B(n-1 ; \alpha)$ sets associated with such sets of directions $S$ will all have Hausdorff dimension at least $n-1+\alpha$. Finally, note that if $n=2$ then the hypothesis that $\mu$ be supported on a curve is no restriction and so all $B(1 ; \alpha)$ sets in $\mathbb{R}^{2}$ have dimension at least $1+\alpha$.

The next result gives, in certain special situations, an improvement over Theorem 1 on the index $q$ in the bound $\left\|R \chi_{E}\right\|_{L_{\mu}^{q, \infty}\left(L^{\infty}\right)} \lesssim|E|^{1 / 2}$.

Proposition 1. Suppose $d \in \mathbb{N}, 1<d<n-1$. Suppose that $\mu$ is the image of Lebesgue measure on a closed ball in $\mathbb{R}^{d}$ under a bi-Lipschitz mapping of that ball into $\Sigma^{(n-1)}$. Then for Borel $E \subseteq B(0,1)$ there is the estimate

$$
\left\|R \chi_{E}\right\|_{L_{\mu}^{2 d, \infty}\left(L^{\infty}\right)} \leq C|E|^{1 / 2}
$$

for some $C=C(n, d, \mu)$.

Proof of Proposition 1. The proof is again analogous to the proof of Theorem 1. The required analogue of Lemma 1 is

Lemma 3. Suppose $\mu$ is as in Proposition 1. Then there is $C$ such that given a Borel $S \subseteq \Sigma^{(n-1)}$ with $\mu(S)>0$ and given $N \in \mathbb{N}$, one can choose $\sigma_{n} \in S, 1 \leq n \leq N$, such that

$$
\sum_{1 \leq m<n \leq N} \frac{1}{\left|\sigma_{m}-\sigma_{n}\right|} \leq \frac{C N^{2}}{\mu(S)^{1 / d}} .
$$

Proof of Lemma 3. Letting $\eta>0$ be defined by $\eta^{d}=\mu(S) /(C N)$, where $C$ is sufficiently large, choose $N \eta$-separated points $\sigma_{1}, \ldots, \sigma_{N}$ from $S$. Then, for fixed $m$,

$$
\sum_{n \neq m} \frac{1}{\left|\sigma_{m}-\sigma_{n}\right|} \lesssim \eta^{-d} \int_{\cup_{n} B\left(\sigma_{n}, \eta / 2\right)} \frac{d \sigma}{\left|\sigma_{m}-\sigma\right|} .
$$

The function $\sigma \mapsto\left|\sigma_{m}-\sigma\right|^{-1}$ is in $L^{d, \infty}(d \mu)$. So, still for fixed $m$,

$$
\sum_{n \neq m} \frac{1}{\left|\sigma_{m}-\sigma_{n}\right|} \lesssim \eta^{-d}\left(N \eta^{d}\right)^{1-1 / d}
$$

The lemma follows from the choice of $\eta$ by summing on $m$.

Returning to the proof of Proposition 1, the analogue of (3) is now

$$
|E| \geq C_{1} N \lambda \delta-C_{2} \delta^{2} N^{2} \mu(S)^{-1 / d},
$$

the choice for $N_{0}$ is $\lambda C_{1} \mu(S)^{1 / d} /\left(C_{2} \delta\right)$, and the remainder of the proof of Proposition 1 is completely parallel to that of Theorem 1 . 


\section{Proof of Theorem 3}

As previously mentioned, the proof is an adaptation of the proof of (3) in [5]. We begin by noting that

$$
\widehat{R f(\sigma, \cdot)}(y)=\int_{-\infty}^{\infty} e^{-2 \pi i y t} \int_{\sigma^{\perp}} f(p+t \sigma) d m_{n-1}(p) d m_{1}(t)=\widehat{f}(y \sigma) .
$$

Thus

$$
\|R f\|_{L_{d \mu}^{2}\left(L^{2}\right)}^{2}=\int_{S} \int_{-\infty}^{\infty}|\widehat{f}(y \sigma)|^{2} d m_{1}(y) d \mu(\sigma)=\int_{\mathbb{R}^{(n-1)}}|\widehat{f}(\xi,|\xi|)|^{2} \frac{d \xi}{|\xi|^{n-2}}
$$

and so estimates for $R$ as a mapping into $L_{\mu}^{2}\left(L^{2}\right)$ are just Fourier restriction estimates for the light cone in $\mathbb{R}^{n}$. More generally, we have

$$
\left\|\left(\frac{\partial}{\partial t}\right)^{\beta} R f\right\|_{L_{\mu}^{2}\left(L^{2}\right)}^{2}=\int_{\mathbb{R}^{(n-1)}}|\widehat{f}(\xi,|\xi|)|^{2} \frac{d \xi}{|\xi|^{n-2-2 \beta}} .
$$

Thus the results of $5.17(\mathrm{~b})$ on p. 367 in [6] give the estimate

$$
\left\|\left(\frac{\partial}{\partial t}\right)^{\beta} R f\right\|_{L_{\mu}^{2}\left(L^{2}\right)} \lesssim\|f\|_{p}
$$

whenever

$$
-\frac{1}{2}<\beta \leq \frac{n-3}{2} \text { and } \frac{1}{p}=\frac{2 n-2 \beta-1}{2 n} .
$$

Estimate (8) will lead to a mixed norm estimate in which the "inside" norm is a Lipschitz norm. The proof of Theorem 3 is simply an interpolation of this estimate with the trivial $L^{1} \rightarrow L^{\infty}\left(L^{1}\right)$ estimate for $R$. The following generalization of an observation from [5] allows this interpolation.

Lemma 4. Fix $\alpha>0$ and $m \in \mathbb{N}$ with $m>\alpha$. For a Borel function $g$ on $\mathbb{R}$ and for $t \in \mathbb{R}$, write $\Delta_{t}$ for the usual difference operator given by $\Delta_{t} g(x)=g(x+t)-g(x), x \in \mathbb{R}$. Let $\|g\|_{\alpha}$ be the Lipschitz norm given by

$$
\|g\|_{\alpha}=\sup _{x \in \mathbb{R}, t \neq 0} \frac{\left|\Delta_{t}^{m} g(x)\right|}{|t|^{\alpha}}
$$

Then, for $1 \leq r<\infty$, we have

$$
\|g\|_{L^{\infty}} \lesssim\|g\|_{L^{r, \infty}}^{\alpha r /(1+\alpha r)}\|g\|_{\alpha}^{1 /(1+\alpha r)}
$$


Proof of Lemma 4. Write

$$
\Delta_{t}^{m} g(x)=\sum_{j=1}^{m} c_{j} g(x+j t) \pm g(x) .
$$

Assume that $|g(x)| \geq \lambda$ for some fixed $x \in \mathbb{R}$ and some $\lambda>0$. If $|t|$ is so small that

$$
|t|^{\alpha}\|g\|_{\alpha} \leq \frac{\lambda}{2}
$$

then

$$
\left|\sum_{j=1}^{m} c_{j} g(x+j t)\right| \geq \frac{\lambda}{2}
$$

Thus

$$
\frac{\lambda}{2}\left(2\left(\frac{\lambda}{2\|g\|_{\alpha}}\right)^{1 / \alpha}\right)^{1 / r} \leq\left\|\sum_{j=1}^{m} c_{j} g(x+j t)\right\|_{L_{t}^{r, \infty}} \lesssim\|g\|_{L^{r, \infty}}
$$

and so

$$
\lambda \lesssim\|g\|_{L^{r, \infty}}^{\alpha r /(1+\alpha r)}\|g\|_{\alpha}^{1 /(1+\alpha r)}
$$

Since $x \in \mathbb{R}$ and $\lambda \leq|g(x)|$ were arbitrary, the desired inequality follows and the proof of Lemma 4 is complete.

For the remainder of this section, the "outside" norms $\|\cdot\|_{L^{s}}$ will refer to the measure $\mu$ on $S$ while $\|\cdot\|_{p}$ will be the norm on $L^{p}\left(\mathbb{R}^{n}\right)$ (or on $L^{p}(\mathbb{R})$ ) and $\|\cdot\|_{\alpha}$ will be the Lipschitz norm of Lemma 4 . Taking $r=1$ in Lemma 4 gives

$$
\|R f\|_{L^{n-2}\left(L^{\infty}\right)} \lesssim\|\| R f\left\|_{1}^{\alpha /(1+\alpha)}\right\|_{L^{\infty}}\|\| R f\left\|_{\alpha}^{1 /(1+\alpha)}\right\|_{L^{n-2}} .
$$

Since

$$
\|R f(\sigma, \cdot)\|_{1} \leq\|f\|_{1}
$$

for all $\sigma \in \Sigma^{(n-1)},(9)$ gives

$$
\|R f\|_{L^{n-2}\left(L^{\infty}\right)} \lesssim\|f\|_{1}^{\alpha /(1+\alpha)}\|\| R f\left\|_{\alpha}^{1 /(1+\alpha)}\right\|_{L^{n-2}} .
$$

To bound the second term of the RHS of (10), we note that the estimate

$$
\|\| R f\left\|_{\alpha}\right\|_{L^{2}} \lesssim\left\|\left(\frac{\partial}{\partial t}\right)^{1 / 2+\alpha} R f\right\|_{L^{2}\left(L^{2}\right)}
$$

follows from Lemma 1 in [5]. Thus if

$$
\alpha=\frac{n-4}{2} \text { and } \frac{1}{p}=\frac{n-1-\alpha}{n}=\frac{n+2}{2 n},
$$


then (8) with $\beta=1 / 2+\alpha$ yields

$$
\begin{aligned}
\|\| R f\left\|_{\alpha}^{1 /(1+\alpha)}\right\|_{L^{n-2}} & =\|\| R f\left\|_{\alpha}\right\|_{L^{2}}^{1 /(1+\alpha)} \\
& \lesssim\left\|\left(\frac{\partial}{\partial t}\right)^{1 / 2+\alpha} R f\right\|_{L^{2}\left(L^{2}\right)}^{1 /(1+\alpha)} \\
& \lesssim\|f\|_{2 n /(n+2)}^{1 /(1+\alpha)} \\
& =\|f\|_{2 n /(n+2)}^{2 /(n-2)} .
\end{aligned}
$$

With (10), this gives

$$
\|\| R \chi_{E}\left\|_{L^{\infty}}\right\|_{L^{n-2}} \lesssim|E|^{(n-1) / n}
$$

which is the desired result.

\section{Miscellany}

\section{Fourier dimension}

As introduced by Kahane in [2], the Fourier dimension of a compact set $E \subseteq \mathbb{R}^{n}$ is twice the least upper bound of the set of nonnegative $\beta$ 's for which $E$ carries a Borel probability measure $\lambda$ satisfying $|\widehat{\lambda}(\xi)|=o\left(|\xi|^{\beta}\right)$ for large $|\xi|$. It is observed in [2] that the Hausdorff dimension of $E$ is always at least equal to the Fourier dimension of $E$ and is generally strictly larger, since the Hausdorff dimension of $E \subseteq \mathbb{R}^{n}$ does not change if $\mathbb{R}^{n}$ if embedded in $\mathbb{R}^{n+1}$ while the Fourier dimension of $E$ now considered as a subset of $\mathbb{R}^{n+1}$ will be 0 . The next result is an analogue for Fourier dimension of the $n=2$ case of Theorem 2:

Proposition 2. Suppose $\alpha \in(0,1)$ and $S \subseteq \Sigma^{(1)}$ has Hausdorff dimension $\alpha$. Suppose that $E$ is a compact subset of $\mathbb{R}^{2}$ containing a unit line segment in each of the directions $\sigma \in S$. Then the Fourier dimension of $E$ is at least $2 \alpha$.

Since Fourier dimension is generally strictly smaller than Hausdorff dimension, it is not surprising that our lower bound $2 \alpha$ for the Fourier dimension of $E$ is strictly smaller than the lower bound $1+\alpha$ for the Hausdorff dimension of $E$ which follows from Theorem 2. Still, it follows from Proposition 2 that Kakeya sets in $\mathbb{R}^{2}$ have Fourier dimension 2, providing a different proof of the well-known fact that such sets have Hausdorff dimension 2. It would be interesting to have examples, for $\alpha \in(0,1)$, of sets $E$ as in the proposition and having Fourier dimension equal to $2 \alpha$. 
Proof of Proposition 2. The heuristic is simple: for each $\beta<\alpha, S$ carries a Borel probability measure $\mu$ satisfying

$$
\mu(J) \leq C|J|^{\beta}
$$

for intervals $J \subseteq \Sigma^{(1)}$ (where $C$ depends on $\beta$ and $|J|$ denotes the "length" of $J$ ).

For each $\sigma \in S$ find $x_{\sigma} \in \mathbb{R}^{2}$ such that $x_{\sigma}+t \sigma \in E$ if $|t| \leq 1 / 2$. Let $\varphi \in C_{0}^{\infty}([-1 / 2,1 / 2])$ be a nonnegative function with integral 1 and define the measure $\lambda$ on $E$ by

$$
\int_{E} f d \lambda=\int_{S} \int_{-1 / 2}^{1 / 2} f\left(x_{\sigma}+t \sigma\right) \varphi(t) d t d \mu(\sigma)
$$

Then

$$
|\widehat{\lambda}(\xi)| \leq \int_{S}\left|\int_{-1 / 2}^{1 / 2} e^{-2 \pi i \xi \cdot\left(x_{\sigma}+t \sigma\right)} \varphi(t) d t\right| d \mu(\sigma)=\int_{S}|\widehat{\varphi}(\xi \cdot \sigma)| d \mu(\sigma) .
$$

For each $p \in \mathbb{N}$ there is $C(p)$ such that

$$
|\widehat{\varphi}(\xi \cdot \sigma)| \leq \frac{C(p)}{|\xi \cdot \sigma|^{p}}
$$

Thus for any $\xi \in \mathbb{R}^{2}$ there are two intervals $J_{1}, J_{2} \subset \Sigma^{(1)}$ of length $\eta>0$ such that for $\sigma \in \Sigma^{(1)}-\left(J_{1} \cup J_{2}\right)$ we have

$$
|\widehat{\varphi}(\xi \cdot \sigma)| \leq \frac{C(p)}{(|\xi| \eta)^{p}}
$$

With (11) and (13) this leads to

$$
|\widehat{\lambda}(\xi)| \lesssim \eta^{\beta}+\frac{1}{(|\xi| \eta)^{p}}
$$

Optimizing with the choice $\eta=|\xi|^{-p /(\beta+p)}$ then gives

$$
|\widehat{\lambda}(\xi)| \leq C(\beta, p)|\xi|^{-\beta p /(\beta+p)},
$$

and this implies the lower bound $2 \beta p /(\beta+p)$ for the Fourier dimension of $E$. As that bound should hold for $0<\beta<\alpha$ and for $p \in \mathbb{N}$, the desired lower bound $2 \alpha$ follows.

The problem with this heuristic argument lies, of course, in the measurability of the selection $\sigma \mapsto x_{\sigma}$. A standard approximation procedure 
circumvents this: for each $N \in \mathbb{N}$, partition $\Sigma^{(1)}$ into $N$ intervals $J_{1}, \ldots, J_{N}$ of length $2 \pi / N$. Choose (if possible) $\sigma_{n} \in J_{n} \cap S$ and define

$$
\mu_{N}=\sum_{n=1}^{N} \mu\left(J_{n}\right) \delta_{\sigma_{n}} .
$$

Define $\lambda_{N}$ as in (12) but with $\mu$ replaced by $\mu_{N}$. Then the argument above shows that

$$
\left|\widehat{\lambda_{N}}(\xi)\right| \leq C(\beta, p)|\xi|^{-\beta p /(\beta+p)}
$$

for $|\xi| \leq N^{1+\beta / p}$. Thus some weak ${ }^{*}$ limit point $\lambda$ of the sequence $\left\{\lambda_{N}\right\}$ will satisfy (14). This completes the proof of Proposition 2.

\section{Examples of $B(2 ; 1)$ sets}

Recall that $E \subseteq \mathbb{R}^{n}$ is a $B(n-1 ; 1)$ set if there is a compact set $S \subseteq \Sigma^{(n-1)}$ having Hausdorff dimension 1 such that for each $\sigma \in S$ there is a hyperplane orthogonal to $\sigma$ which intersects $E$ in a set of positive $(n-1)$-dimensional Lebesgue measure. Although we have not proved it unless $S$ sits on a nice curve in $\Sigma^{(n-1)}$, one expects that $B(n-1 ; 1)$ sets should have Hausdorff dimension $n$. Here are some examples in dimension 3 :

Example 1. Suppose that $\widetilde{E}$ is a (Kakeya) subset of $\mathbb{R}^{2} \hookrightarrow \mathbb{R}^{3}$ having 2-dimensional Lebesgue measure 0 and containing a line segment in each direction. If $E$ is the product of $\widetilde{E}$ and a line segment orthogonal to $\mathbb{R}^{2}$, then $E$ is a measure-zero $B(2 ; 1)$ set having full dimension and associated with the 1-sphere of directions

$$
S_{1} \doteq\left\{\sigma=\left(\sigma_{1}, \sigma_{2}, \sigma_{3}\right) \in \Sigma^{(2)}: \sigma_{3}=0\right\} .
$$

Example 2. Suppose that $S \subseteq \Sigma^{(2)}$ is a compact set of Hausdorff dimension 1 which supports a Borel probability measure $\mu$ satisfying the condition

$$
\int_{S} \int_{S} \frac{d \mu\left(\sigma_{1}\right) d \mu\left(\sigma_{2}\right)}{\left|\sigma_{1}-\sigma_{2}\right|}<\infty
$$

(It is not too difficult to construct such an $S$ and $\mu$ using a Cantor set with variable ratio of dissection.) The proof of Theorem 1 yields in this case the estimate

$$
\left\|R \chi_{E}\right\|_{L_{\mu}^{1, \infty}\left(L^{\infty}\right)} \lesssim|E|^{1 / 2}
$$

for Borel $E \subseteq B(0,1)$. Thus any $B(2 ; 1)$ set associated with the set of directions $S$ must have not only full dimension but also positive measure. 
Example 3. Consider the 1-sphere of directions

$$
S_{2} \doteq\left\{\sigma=\left(\sigma_{1}, \sigma_{2}, \sigma_{3}\right) \in \Sigma^{(2)}: \sigma_{1}^{2}+\sigma_{2}^{2}=\sigma_{3}^{2}\right\} .
$$

As with $S_{1}$ in Example 1, it follows from Theorem 2 that the $B(2 ; 1)$ sets associated with $S_{2}$ have full dimension. A difference between $S_{1}$ and $S_{2}$ appears when considering the possibility of

$$
L^{p} \rightarrow L_{\mu_{j}}^{2}\left(L^{2}\right)
$$

estimates for $R$ (here $\mu_{j}$ is Lebesgue measure on the circle $S_{j}$ ). For $j=2$ there will be such an estimate for $p=6 / 5$. This follows from (8) and, as mentioned in the proof of Theorem 3, is just the Tomas-Stein restriction theorem for the light cone in $\mathbb{R}^{3}$. On the other hand, there is no estimate (15) for $\mu_{1}$ (because there are no Fourier restriction theorems for hyperplanes). It would be interesting to know whether, in contrast to the situation in Example 1 , the $B(2 ; 1)$ sets associated with $S_{2}$ must actually have positive measure.

\section{Unions of collections of hyperplanes}

The ideas in the proofs of Theorems 1 and 2 can be used to give some answers to special cases of the following question: if $\mathcal{P}$ is a collection of hyperplanes, what can be said about the size of

$$
\bigcup_{P \in \mathcal{P}} P
$$

given information about the size of $\mathcal{P}$ ? To illustrate, we will consider one case by indicating why (16) must have positive measure if the dimension of $\mathcal{P}$ exceeds 1. Parametrize the set of hyperplanes in $\mathbb{R}^{n}$ as $\Sigma^{(n-1)} \times[0, \infty)$ by writing $P=(\sigma, t)$ if $P=\sigma^{\perp}+t \sigma$ and say that a compact set $\mathcal{P}$ of hyperplanes has dimension $\alpha>0$ if, for each $\epsilon \in(0, \alpha), \mathcal{P}$ carries a Borel probability measure $\mu$ such that

$$
\int_{\mathcal{P}} \int_{\mathcal{P}} \frac{d \mu\left(P_{1}\right) d \mu\left(P_{2}\right)}{\left(\left|\sigma_{1}-\sigma_{2}\right|+\left|t_{1}-t_{2}\right|\right)^{\alpha-\epsilon}}<\infty
$$

Fix such a $\mathcal{P}$ and $\mu$. Writing $P_{\sigma, t}^{\delta}$ for the plate $\left[\sigma^{\perp} \cap B(0,1)\right]+B(0, \delta)+t \sigma$, one can check that if

$$
P_{\sigma_{1}, t_{1}}^{\delta} \cap P_{\sigma_{2}, t_{2}}^{\delta} \neq \emptyset
$$

then $\left|t_{1}-t_{2}\right| \lesssim\left|\sigma_{1}-\sigma_{2}\right|+\delta$. This leads to the bound

$$
\left|P_{\sigma_{1}, t_{1}}^{\delta} \cap P_{\sigma_{2}, t_{2}}^{\delta}\right| \leq \frac{C(n) \delta^{2}}{\left|\sigma_{1}-\sigma_{2}\right|+\left|t_{1}-t_{2}\right|}
$$


if $\sigma_{1}$ and $\sigma_{2}$ are not too far apart. Let $R_{0}$ be the truncated Radon transform given by

$$
R_{0} f(\sigma, t)=\int_{\sigma^{\perp} \cap B(0,1)} f(p+t \sigma) d m_{n-1}(p) .
$$

If $\alpha-\epsilon>1$, the proof of Theorem 1 now gives the estimate

$$
\left\|R_{0} \chi_{E}\right\|_{L_{\mu}^{\alpha-\epsilon, \infty}} \lesssim|E|^{1 / 2}
$$

for Borel $E \subseteq \mathbb{R}^{n}$. It follows that if

$$
\bigcup_{P \in \mathcal{P}} P \subseteq E
$$

then $|E|>0$.

\section{References}

[1] Bourgain, J.: Besicovitch type maximal operators and applications to Fourier analysis. Geom. Funct. Anal. 1 (1991), 147-187.

[2] Kahane, J.-P.: Some Random Series of Functions. Cambridge Studies in Advanced Mathematics, 5. Cambridge University Press, 1993.

[3] Oberlin, D. M.: $L^{p} \rightarrow L^{q}$ mapping properties of the Radon transform. In Banach spaces, harmonic analysis, and probability theory (Storrs, Conn., 1980/1981), 95-102. Lecture Notes in Math., 995. Springer, Berlin, 1983.

[4] Oberlin, D. M.: An estimate for a restricted X-ray transform. Canad. Math. Bull. 43 (2000), 472-476.

[5] Oberlin, D. M. And Stein, E. M.: Mapping properties of the Radon transform. Indiana Univ. Math. J. 31 (1982), 641-650.

[6] Stein, E. M.: Harmonic analysis: real-variable methods, orthogonality, and oscillatory integrals. Princeton Mathematical Series 43. Monographs in Harmonic Analysis, III. Princeton University Press, Princeton, NJ, 1993.

Recibido: 4 de enero de 2005

Revisado: 28 de febrero 2005

Daniel M. Oberlin

Department of Mathematics

Florida State University

Tallahassee, FL 32306-4510

oberlin@math.fsu.edu 